\title{
H-INDEX CONCATENATION
}

\author{
WOLFGANG GLÄNZEL ${ }^{\mathrm{a}, \mathrm{b}}$ \\ ${ }^{a}$ K.U. Leuven, Steunpunt O\&O Indicatoren, Dept. MSI, Leuven, Belgium \\ ${ }^{b}$ Hungarian Academy of Sciences, IRPS, Budapest, Hungary
}

\begin{abstract}
A method for the calculation of a 'concatenated' h-index of jointly ranked combined bibliographies is presented in the case when only size and h-index of the original publication sets are known.
\end{abstract}

\section{INTRODUCTION}

Despite all attempts to refine, adjust or even improve the h-index, Hirsch's original measure prevailed, and has already become a standard tool in evaluative bibliometrics. However, handling of this simple but somewhat uncommon statistic proved rather difficult. While classical statistics has comprehensive answers to the questions of how to treat and evaluate shares, means or extreme values, the problem of comparing or combining different $h$-indexes is still unsolved. Nonetheless, the question of how the h-index of the combination of two bibliographies relates to their original h-indexes is certainly of interest.

Re-ranking the union of two bibliographies is practically the only way to determine their joint $\mathrm{h}$-index. The question, of course, arises of whether it is possible to directly derive the joint $\mathrm{h}$-index from different individual samples without creating and re-ranking the combined publication set. In other words, how can we calculate the concatenated $h$-index of two or more $h$-indexes if besides the h-indexes no further information is available?

\section{THE CONCATENATED H-INDEX}

In order to solve this problem, we assume that the samples are based on independent, identically distributed random variables and the samples are disjoint. Analogously to earlier papers by the author (Glänzel, 2006, 2008), it is furthermore assumed for simplicity's sake that the common citation distribution can be approximated by a non-negative continuous distribution. In particular, a Lomax distribution (a Pareto distribution of the second kind) is proposed, that is, if $X$ represents the citation rate of a paper then

$$
G(x):=P(X \geq x)=\frac{N^{\alpha}}{(N+x)^{\alpha}}, \text { for all } x \geq 0,
$$

where $N>0$ and $\alpha>0$ are the two free parameters of the Lomax distribution. If $x$ is large $(x \gg N)$, the parameter in the denominator can be neglected and we have

$$
G(x) \approx N^{\alpha} / x^{\alpha}, \text { for } x \gg N .
$$

The final publication is available at Springer via http://dx.doi.org/10.1007/s11192-008-2104-x 
We assume a sample of $n$ observations $\left\{X_{i}\right\}_{i=1, \ldots, n}$, where all elements are independent and have a Lomax distribution with the same parameters $N$ and $\alpha$. In an earlier paper (Glänzel, 2008) it was shown that under these conditions the following equality holds.

$$
h=N^{\alpha /(\alpha+1)} \cdot n^{1 /(\alpha+1)} \Leftrightarrow h^{\alpha+1}=N^{\alpha} \cdot n .
$$

Now we assume that we have two disjoint samples $\left\{X_{i}\right\}$ and $\left\{X_{j}\right\}$ of size $n^{\prime}$ and $n^{\prime \prime}$, respectively. We also assume that the elements of both samples are independent and identically distributed. The h-indexes associated with the two samples are $h^{\prime}$ and $h^{\prime \prime}$. In the following the concatenated $h$-index $\left(h^{\prime} \circ h\right)$ of the joint ranking list of the combined sample $\left\{X_{i}\right\}_{i=1, \ldots, n^{\prime}, n^{\prime}+1, \ldots, n^{\prime}+n^{\prime \prime}}$ of size $n:=n^{\prime}+n^{\prime \prime}$ will be determined. Applying Eq. 2 to the two samples results in $h^{\prime(\alpha+1)}=N^{\alpha} n^{\prime}$ and $h^{\prime \prime(\alpha+1)}=N^{\alpha} n^{\prime \prime}$ with $h^{\prime(\alpha+1)}+h^{\prime \prime(\alpha+1)}=N^{\alpha} \cdot n^{\prime}+N^{\alpha} \cdot n^{\prime \prime}=N^{\alpha}\left(n^{\prime}+n^{\prime \prime}\right)=N^{\alpha} n=h^{(\alpha+1)}$. Hence we have

$$
h=h^{\prime} \circ h^{\prime \prime}=\left\{h^{\prime(\alpha+1)}+h^{\prime \prime(\alpha+1)}\right\}^{1 /(\alpha+1)} .
$$

Repeated application of concatenation results in the following theorem.

Theorem 1. Assume that the random variables of $k$ disjoint samples $\left\{X_{i j}\right\}_{j=1, \ldots, n_{i}}$ of size $n_{i}(i=$ $1,2, \ldots, k)$ are independent and are identically distributed. The common distribution is Lomax with parameters $N>0$ and $\alpha>0$. Assume further that the $h$-index of each sample is $h_{i}$. Then the concatenated $h$-index $\left(\circ h_{i}\right)$ of the joint sample $\left\{X_{j}\right\}_{j=1, \ldots, \sum n_{i}}$ is obtained from the $h$-indexes of the original samples $h_{i}$ in the following manner.

$$
h=\left(\circ h_{i}\right)=h_{1} \circ h_{2} \circ \ldots \circ h_{k}=\left\{\sum_{i=1}^{k} h_{i}^{(\alpha+1)}\right\}^{1 /(\alpha+1)} .
$$

Remark 1. This approximation holds even if $n_{i}=1$ for all $i=1,2, \ldots, k$, provided $N \ll 1$. In this case we have $h_{i}=1$, if the paper is cited, and $h_{i}=0$, otherwise. The case $h_{i}=1$ occurs with probability $P(X \geq 1)=N /(N+1)$ This results in the following approximation if $N \ll 1$.

$$
\left\{\sum_{i=1}^{k} h_{i}^{(\alpha+1)}\right\}=\frac{N^{\alpha} \cdot n}{(N+1)^{\alpha}} \approx N^{\alpha} \cdot n=h^{(\alpha+1)} .
$$

Remark 2. $h$ takes integer values; therefore $\left\{\sum_{i} h_{i}^{(\alpha+1)}\right\}^{1 /(\alpha+1)}$ has to be rounded.

Remark 3. Let $h=\max \left(h_{1}, h_{2}\right)$ be the maximum of two $h$-indexes. Then the following inequality always holds. $h \leq h_{1} \circ h_{2} \leq \sqrt[\alpha+1]{2} \cdot h$. The lower limit is taken if one of the $h$-indexes is $0 ; h$ takes the upper limit if $h_{1}=h_{2}$. For $\alpha=2$ this amounts to about $1.26 \cdot h$.

Example 2.1. In earlier papers (Schubert and Glänzel, 2008, Glänzel, 2008), it was shown that the assumption of $\alpha \approx 2$ provides good results for short citation windows comprising an initial period of about three years after publication. In 2002, the two multidisciplinary journals Science and Nature had an h-index of $h_{1}=106$ and $h_{2}=108$, respectively, if a three-year citation window 
TABLE 1. h-index of the journals Science (A) and Nature (B) and the h-index of the jointly ranked publication list of both journals (publication year: 2002, citation window: 2002-2004)

\begin{tabular}{cccccc}
\hline & \multicolumn{1}{l}{$\mathbf{A}$} & \multicolumn{2}{c}{$\mathbf{B} \cup \mathbf{B}$} \\
$\boldsymbol{r}$ & $\boldsymbol{R}(\boldsymbol{r})$ & $\boldsymbol{r}$ & $\boldsymbol{R}(\boldsymbol{r})$ & $\boldsymbol{r}$ & $\boldsymbol{R}(\boldsymbol{r})$ \\
\hline 97 & 112 & 99 & 113 & 125 & 140 \\
98 & 111 & 100 & 112 & 126 & 140 \\
99 & 111 & 101 & 111 & 127 & 140 \\
100 & 111 & 102 & 110 & 128 & 139 \\
101 & 110 & 103 & 110 & 129 & 138 \\
102 & 110 & 104 & 109 & 130 & 137 \\
103 & 110 & 105 & 109 & 131 & 136 \\
104 & 109 & 106 & 109 & 132 & 135 \\
105 & 106 & 107 & 108 & 133 & 134 \\
$\mathbf{1 0 6}$ & $\mathbf{1 0 6}$ & $\mathbf{1 0 8}$ & $\mathbf{1 0 8}$ & $\mathbf{1 3 4}$ & $\mathbf{1 3 4}$ \\
107 & 106 & 109 & 108 & 135 & 134 \\
\hline
\end{tabular}

is applied. According to Theorem 1 we obtain $h=h_{1} \circ h_{2}=\sqrt[3]{h_{1}^{3}+h_{2}^{3}}=\sqrt[3]{1063+1083}=134.8$ for the joint ranking list of the combined sample. The empirical value amounts to $h=134$. Table 1 presents the ranks $(r)$ around the h-index as well as the citation rates of the $r$ th most frequently cited papers $(R(r))$ for both journals as well as for the combined publication list.

TABLE 2. h-index of two bibliometricians and the h-index of the jointly ranked publication list of both authors (Data retrieved in March 2008)

\begin{tabular}{cccccc}
\hline \multicolumn{2}{c}{$\mathbf{A}$} & \multicolumn{2}{c}{$\mathbf{B}$} & \multicolumn{2}{c}{$\mathbf{A} \cup \mathbf{B}$} \\
$\boldsymbol{r}$ & $\boldsymbol{R}(\boldsymbol{r})$ & $\boldsymbol{r}$ & $\boldsymbol{R}(\boldsymbol{r})$ & $\boldsymbol{r}$ & $\boldsymbol{R}(\boldsymbol{r})$ \\
\hline 3 & 23 & 14 & 27 & 15 & 27 \\
4 & 22 & 15 & 26 & 16 & 27 \\
5 & 21 & 16 & 25 & 17 & 26 \\
6 & 17 & 17 & 24 & 18 & 25 \\
7 & 17 & 18 & 23 & 19 & 24 \\
8 & 16 & 19 & 23 & 20 & 23 \\
9 & 15 & 20 & 23 & 21 & 23 \\
10 & 15 & 21 & 23 & 22 & 23 \\
$\mathbf{1 1}$ & $\mathbf{1 4}$ & $\mathbf{2 2}$ & $\mathbf{2 2}$ & $\mathbf{2 3}$ & $\mathbf{2 3}$ \\
12 & 8 & 23 & 22 & 24 & 23 \\
13 & 8 & 24 & 21 & 25 & 22 \\
\hline
\end{tabular}

Example 2.2. In contrast to the previous example, I have selected two bibliographies with distinctly different h-indexes. This example refers to Hirsch's original idea of ranking citation rates of individuals. I have selected two bibliometricians who have never co-authored papers. 
This guarantees that the two bibliographies are disjoint. Only articles, letters, notes and reviews relevant for the field of bibliometrics are taken into account. Since the intention of this example is merely the illustration of the application of Theorem 1, and not the evaluation of individual scientists, the two authors are treated anonymously. The h-index of author A amounts to 11; that of author B is 22. Table 2 presents the ranks and citation rates for the authors in the same way as Table 1 . The concatenated h-index of the two authors is 23 .

Unlike in the previous case, citations are cumulated from the publication year till 2008 . Both authors are active since the 1980s. Although $\alpha$ might therefore possibly differ from 2 , the assumption of $\alpha=2$ provides acceptable results. According to Theorem 1, we have $h=h_{1} \circ h_{2}=$ $\sqrt[3]{h_{1}^{3}+h_{2}^{3}}=\sqrt[3]{113+223}=22.9$. This again illustrates the robustness of the formula for Hirschindex concatenation.

\section{REFERENCES}

Glänzel, W. (2006), On the H-index - A mathematical approach to a new measure of publication activity and citation impact, Scientometrics, 67 (2), 315-321.

Glänzel, W. (2008), On some new bibliometric applications of statistics related to the h-index, Scientometrics, 76 (3), forthcoming.

Schubert, A., Glänzel, W. (2007), A systematic analysis of Hirsch-type indices for journals. Journal of Informetrics, 1 (3), 179-184. 